\title{
Numerical Nonlinear Analysis with the Boundary Element Method
}

\author{
E. Pineda ${ }^{1}$, I. Villaseñor ${ }^{1}$ and J. Zapata ${ }^{1}$ \\ ${ }^{1}$ Instituto Politécnico Nacional, ESIA-UZ, Unidad Profesional Adolfo López Mateos \\ México D.F. (epinedal@ipn.mx)
}

\begin{abstract}
This paper presents a new formulation of the Boundary Element Method to viscoplastic problems in a two-dimensional analysis. Visco-plastic stresses and strains are obtained until the visco-plastic strain rate reaches the steady state condition. A perfect viscoplastic analysis is also carried out in linear strain hardening $\left(H^{\prime}=0\right)$ materials. Part of the domain, the part that is susceptible to yield is discretized into quadratic, quadrilateral continuous cells. The loads are used to demonstrate time effects in the analysis carried out. Numerical results are compared with solution obtained from the Finite Element Method (FEM).
\end{abstract}

Keywords: Boundary Element, Visco-plasticity, Boundary Integral Equations

\section{INTRODUCTION}

In the case of problems with high temperature gradients where inelastic strain rates are proportional to high power of stress, regions with strain rate concentration provide nearly all the inelastic contribution to the stress rates [1]. The main reason for the success of the BEM (boundary element method) in any problem is the ability to model high stress concentration fields accurately and efficiently. A comprehensive review of the historic development of the BEM for inelasticity can be found in the work of Aliabadi [2]. An alternative methodology based on the use of the Kelvin fundamental solutions was presented in [3], [4] and[5]. Recently, the DBEM (dual boundary element method) has been developed as a very effective numerical tool to model general fracture problems with numerous applications to linear elastic and non-elastic fracture problems [6].

BEM has been applied to elastoplastic problems since the early seventies with the work of Swedlow and Cruse [7] and Richardella [8] who implemented the von Mises criterion for 2D problems using piecewise constant interpolation for the plastic strains. Later, Telles and Brebbia [9] and others had, by the beginning of the eighties, developed and implemented BEM formulations for 2D and 3D inelastic, viscoplastic and elastoplastic problems (see [10] for further details).

In recent years, Aliabadi and co-workers [11] have introduced a new generation of boundary element method for solution of fracture mechanics problems. The method which was originally proposed for linear elastic problems[12], [13] and [14] has since been extended to many other fields including problems involving nonlinear material and geometric behavior [15]. 
In the present paper applications of the DBEM to visco-plasticity are presented. The specimens analyzed are three different plates. The boundary was discretized with quadratic continuous and semi-discontinuous elements, but the domain with nine nodes internal cells. In visco-plasticity only the part susceptible to yielding was discretized. The von Mises yield criterion was applied so the material used for these sort of analysis were metals.

\section{VISCO-PLASTICITY THEORY}

In order to explain the theory of visco-plasticity it is convenient to analyze the onedimensional rheological model see ¡Error! No se encuentra el origen de la referencia. for more details. A uniaxial yield stress $\sigma_{y}$ governs the onset of the visco-plastic deformation. Once visco-plasticty begins the stress level for continuing visco-plastic flow depends on the strain hardening characteristics of the material $\left(H^{\prime} \neq 0\right)$.

After applying Hook's law and boundary conditions, it is possible to obtain:

$$
\dot{\varepsilon}^{v p}=\gamma\left[\sigma-\left(\sigma_{y}+H^{\prime} \varepsilon^{v p}\right)\right]
$$

Expression (1) is the visco-plastic strain rate in terms of the stresses for the uniaxial case in which (.) denotes the derivative with respect to the time, $t$.

From the visco-plastic model the strain response with time can be represented by two cases. The first case is the perfectly visco-plastic material in which $H^{\prime}=0$. In this case the viscoplastic deformation continues at a constant strain rate.

The second case is the linear hardening case $\left(H^{\prime} \neq 0\right)$, where after the initial elastic response, the visco-plastic strain rate is exponential and reaches the steady state condition when this value becomes zero. On the other hand, for a perfectly visco-plastic material there is always an imbalance of stress $\sigma_{a}-\sigma_{y}$ in the system which does not reduce and consequently the steady state condition can not be achieved.

\section{BOUNDARY INTEGRAL EQUATIONS}

The boundary conditions in terms of rates are; for displacements $\dot{u}_{i}=\ddot{u}_{i}$ and for tractions $\dot{t}_{i}=\ddot{t}_{i}$ and the equation representing the traction boundary conditions is,

$$
\dot{t}_{i}+2 \mu\left(\dot{\varepsilon}_{i j}{ }^{a}+\frac{v}{1-2 v} \dot{e}\right) n_{j}=\frac{2 \mu \nu}{1-2 v} \dot{u}_{l, l} n_{i}+\mu\left(\dot{u}_{i, j}+\dot{u}_{j, i}\right) n_{j}
$$

Equation (2) is for three dimensional problems. In order to work with two dimensional problems for the plane stress state it is necessary to remove the strain in z direction, so $\varepsilon^{a}{ }_{33}=0$.

The solution of the equation (2) leads to the following boundary Integral representation of the boundary displacements when the initial strain approach for the solution of inelastic problems is used 


$$
c_{i j} \dot{u}_{i}+\int_{\Gamma} \dot{t}_{i j}^{\prime} \dot{u}_{j} d \Gamma=\int_{\Gamma} \dot{u}_{i j}^{\prime} \dot{t}_{j} d \Gamma+\int_{\Omega} \sigma_{i j k}^{\prime} \dot{\varepsilon}^{p}{ }_{j k} d \Omega
$$

In a similar way the boundary integral equation of the internal stresses is expressed by

$$
\sigma_{i j}=\int_{\Gamma} D_{i j k} t_{j} d \Gamma-\int_{\Gamma} S_{i j k} \dot{u}_{j} d \Gamma+\int_{\Omega} \Sigma_{i j k} \dot{\varepsilon}^{a}{ }_{j k} d \Omega+f_{i j} \dot{\varepsilon}^{p}{ }_{j k}
$$

Where $\oint$ is a Cauchy integral, $D_{i j k}$ and $S_{i j k}$ are terms containing the derivative of the displacements and tractions, $f_{i j}$ is the free term and $\Sigma_{i j}$ is the fundamental solution for the domain.

\subsection{Boundary Integral Formulation for Visco-plasticity}

In the visco-plastic analysis like plasticity, the initial strain approach will be applied and the integral equation to calculate the displacement on the boundary is basically the same, the only difference is that the plastic strain is replaced with the visco-plastic strain rate. So the displacement equation can be rewritten as:

$$
c_{i j}\left(x^{\prime}\right) \dot{u}_{j}\left(x^{\prime}\right)+\int_{\Gamma} t_{i j}^{\prime}\left(x^{\prime}, x\right) \dot{u}_{i}\left(x^{\prime}\right) d \Gamma=\int_{\Gamma} u_{i j}^{\prime}\left(x^{\prime}, x\right) \dot{t}_{j}\left(x^{\prime}\right) d \Gamma+\int_{\Omega} \sigma_{i j}^{\prime}\left(x^{\prime}, z\right) \dot{\varepsilon}^{v p}{ }_{i j}(z) d \Omega
$$

Where $\dot{u}_{i}, \dot{t}_{i}$ and $\dot{\varepsilon}^{v p}{ }_{i j}$ are the displacement, traction and visco-plastic strain rates respectively. $t_{i j}^{\prime}, u_{i j}^{\prime}$ and $\sigma_{i j}^{\prime}$ are the displacement, traction and third order fundamental solutions, respectively, which are functions of the positions of the collocation point $\mathrm{x}^{\prime}$ and the field point $\mathrm{x}$ which belong to the boundary, or the internal point $\mathrm{z}$ and the material properties.

In order to illustrate the results obtained with the Boundary Element Method three different plates were analyzed as is shown below.

\section{EXAMPLES}

\subsection{The Notched plate}

An aluminum plate with a notch and geometry as illustrated in figure 1, it is considered in this case. The plate is constrained in $\mathrm{X}$ and $\mathrm{Y}$ direction on the edge of the notch and it is assumed to have the following material properties: Young's modulus, $\mathrm{E}=70000 \mathrm{MPa}$; Poisson's ratio $\nu=0.2$; Applied stress $\sigma_{a}=140 \mathrm{Mpa} ., \sigma_{y}=243 \mathrm{Mpa}$. with $\gamma=0.01$ and $\Delta t=0.01 \mathrm{~s}$ 


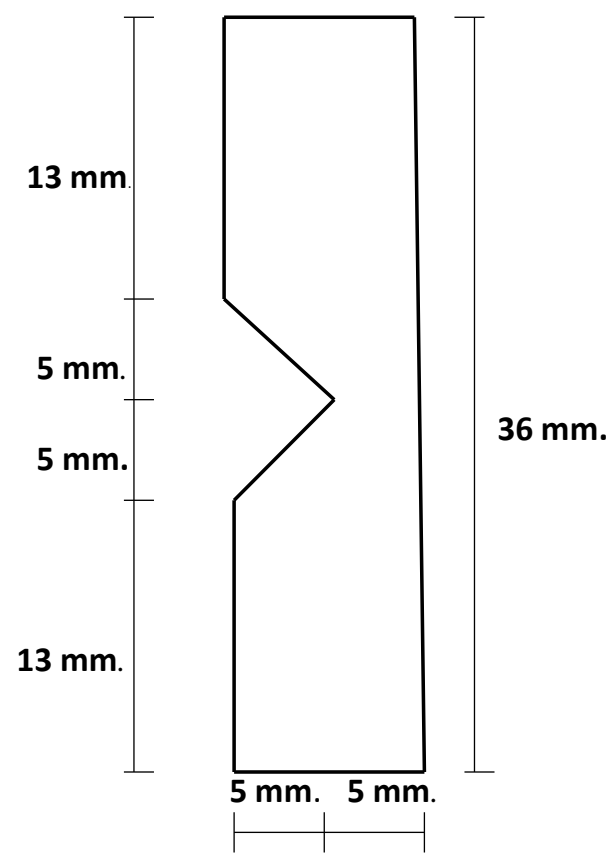

Figure 1 Geometry in a plate with a notch

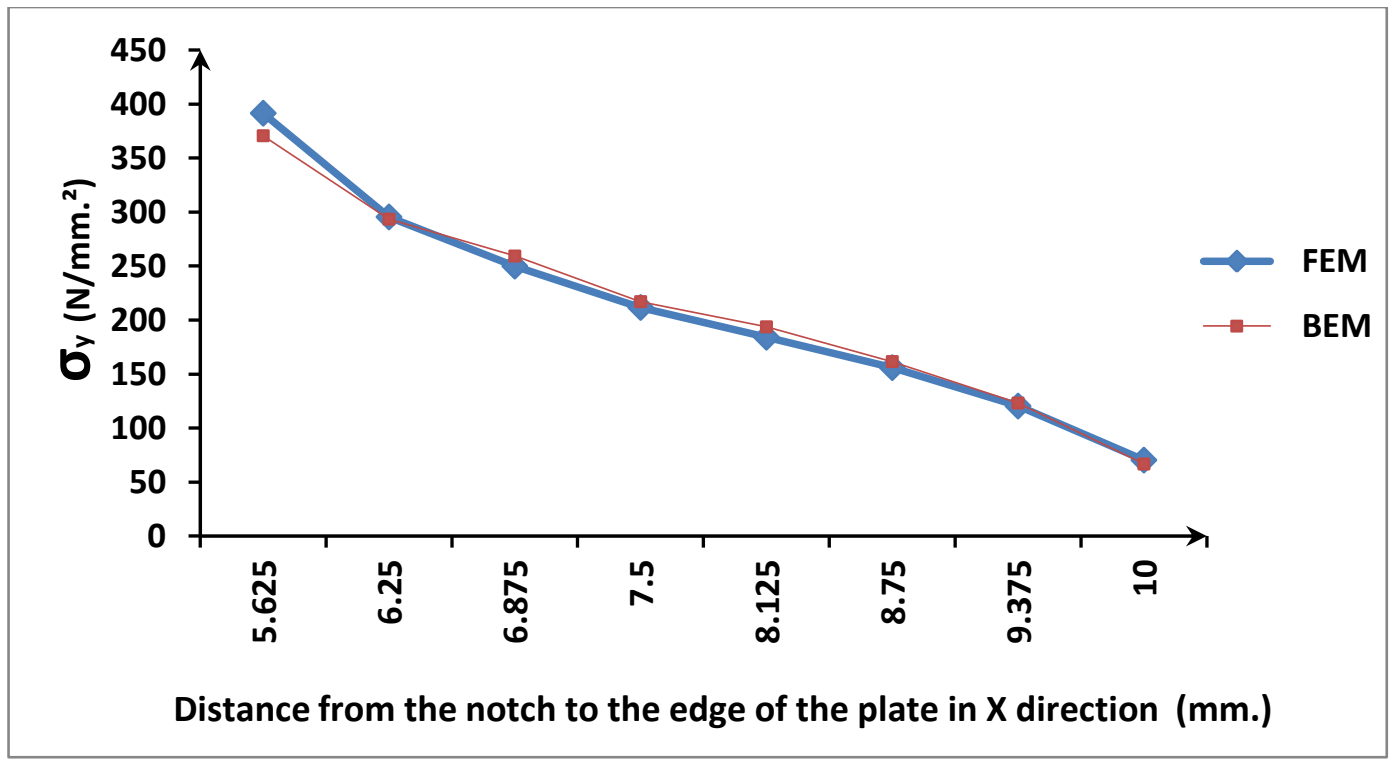

Figure 2. Stresses in Y direction for a notched plate. 


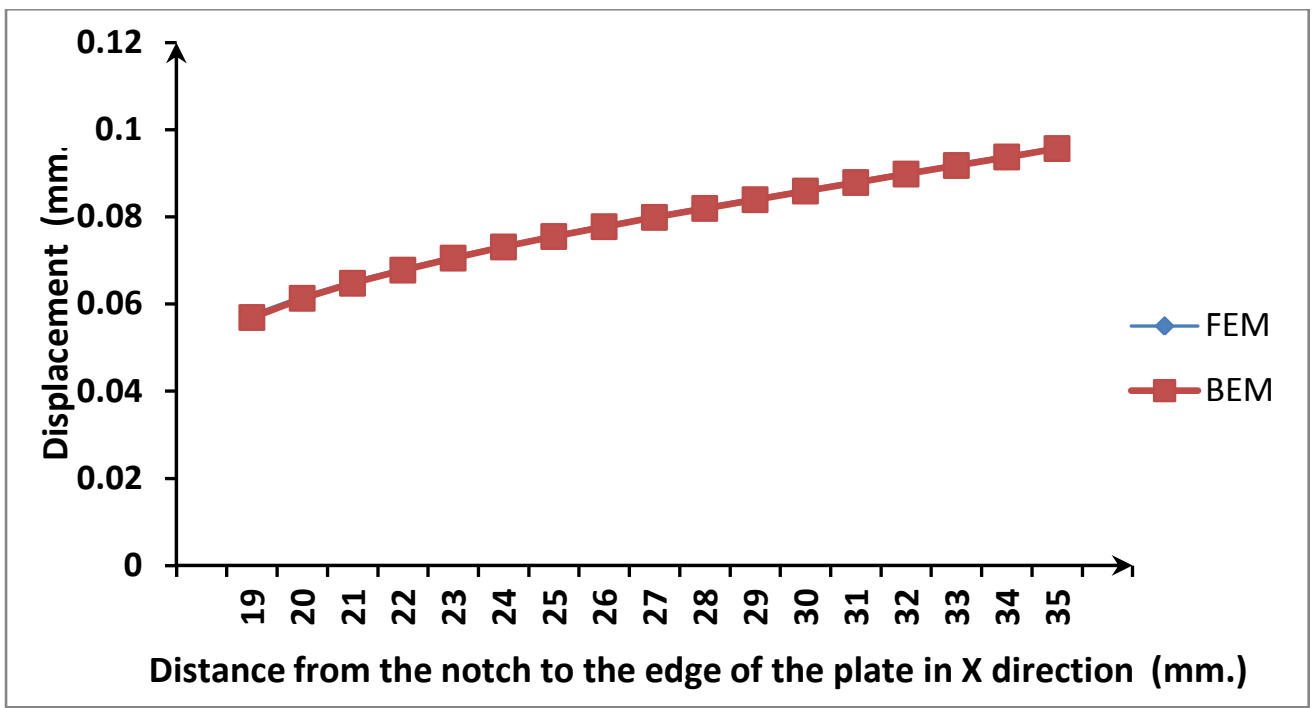

Figure 3. Displacements in Y direction for a notched plate.

\subsection{Plate with a hole}

A perforated tension specimen with dimensions and geometry as illustrated in figure 4, it is considered in this example. The plate is constrained in $\mathrm{X}$ and $\mathrm{Y}$ direction on the edge and was subjected to the same tensile load, material properties and constants as the example 4.1.

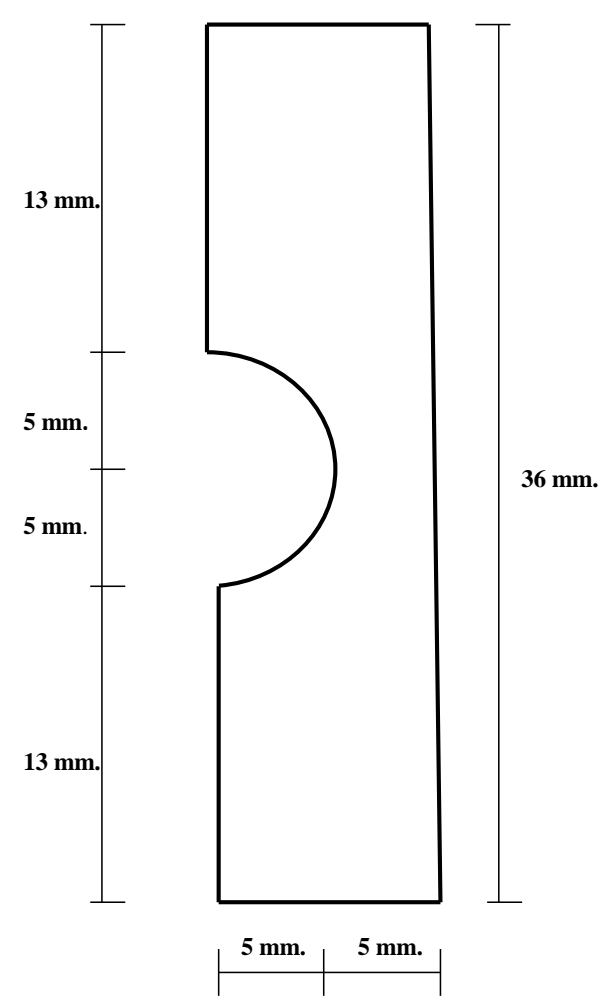


Figure 4 Geometry for a plate with a hole

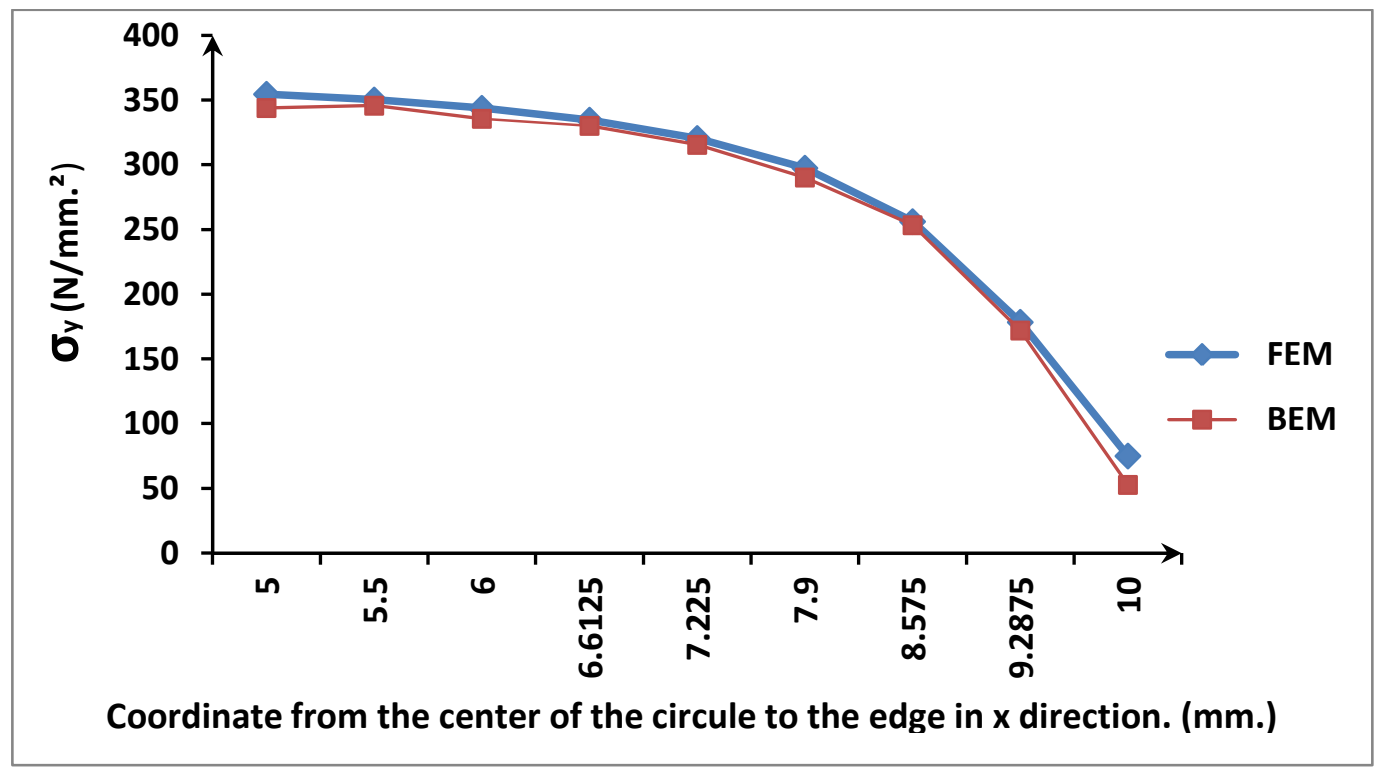

Figure 5 Stresses in the center of the plate in Y direction from the center of the hole.

\section{CONCLUSIONS}

In this paper the BEM was applied to the analysis of non-elastic time dependent problems. It has been demonstrated here that this method is an accurate and efficient method for analyzing and modeling visco-plastic problems. The visco-plastic stresses and displacements obtained of the Boundary Element Method are in good agreement with the ones calculated in the Finite Element Method. The increment of time plays a very important role for the accuracy of the results, if we make a good selection the efficiency of the program and the results will be guaranteed. The visco-plastic behaviour is represented by a plastic strain field over a region, susceptible to yield, discretized with quadrilateral quadratic continuous and discontinuous internal cells.

\section{REFERENCES}

[1] Providakis, C.P., Viscoplastic BEM Fracture Analysis of Creeping Metallic Cracked Structures in Plane Stress using Complex Variable Technique. Engineering Fracture Mechanics, 70, 707-720.,2003

[2] Aliabadi, M.H., Boundary Element Methods in Fracture Mechanics. Appl Mech Rev, , 50, 83-96.,1997

[3] Tan, C.L., Lee, K.H. Elastic-Plastic Stress Analysis of a Cracked thick-walled Cylinder, Journal of Strain Analysis, 50-57., 1983

[4] Yong, L., and Guo, W.G., The calculation of $J_{I}$ Integrals of Thick-Walled Tubes with One and Two Symmetrick Cracks by Elastoplastic BEM, Int. J. Pres. Ves. \& Pipping, 51, 143-154. 1992

[5] Hantschel T., Busch, M., Kuna, M., and Maschke, H.G., Solution of Elastic-Plastic Crack Problems by an Advanced Boundary Element Method, in Proceedings of the 5th. International Conference on Numerical Methods in Fracture Mechanics, A.R.. 
Luxmoore and D.R.J. Owen, Eds., pp. 29-40., 1990

[6] Leitão, V., An Improved Bounadry Element Formulation for Nonlinear Fracture Mechanics I, PhD Thesis, Wessex Institute of Technology, University of Porsmouth, 1993.

[7] Swedlow, J. L. and Cruse, T. A. Formulation of the boundary integral equation for three-dimensional elastoplastic flow, International Journal of Solids and Structures, 7, 1673-1681., 1971

[8] Riccardella, P. An Implementation of the Boundary Integral Technique for plane problems of Elasticity and Elastoplasticity, PhD Thesis, Carnegie Mellon University, Pitsburg, PA 1973.

[9] Telles, J. C. F., and Brebbia,C.A. Elastic/viscoplastic Problems using Boundary Elements,International Journal of Mechanical Sciences, 24, 605-618. 1982

[10] Aliabadi, M.H., The Boundary Element Method. Applications in Solids and Structures. Vol. 2. John Wiley \& Sons, Ltd, West Sussex, England 2002.

[11] Aliabadi,M.H. A new generation of boundary element methods in fracture mechanics,International Journal of Fracture, 86, 91-125. 1997

[12] Aliabadi,M.H. and Portela,A. Dual boundary element incremental analysis of crack growth in rotating disc. Boundary Element Technology VII, Computational Mechanics Publica-tions, Southampton, 607-616. 1992

[13] Portela, A. Aliabadi, M.H., and Rooke, D.P., Dual Boundary Element Incremental Analysis of Crack propagation. Computers \& Structures, 46, 237-247. 1993

[14] Mi, Y., Aliabadi, M.H., Dual Boundary Element Method for Three-Dimensional Fracture Mechanics Analysis, Engng. Anal. with Bound. Elem., 10, 161-171. 1992

[15] Chao Y.J., Zhu, X.K., and Zhang, L., Higher-Order Asymptotic Crack-Tip .elds in a power-lawCreeping material, International Journal of Solids and Structures, 38, 2001.

[16] Pineda, E., Dual Boundary Element Analysis for Creep Fracture, Ph. D. Thesis, Queen Mary College, University of London, 2005. 